\title{
Jaw-thrust induces sympathetic responses during induction of general anesthesia
}

\author{
Sang-Jin Park, Bum Soo Kim, and Dae-Lim Jee \\ Department of Anesthesiology and Pain Medicine, Yeungnam University Hospital, Daegu, Korea
}

Background: Jaw-thrust is a noxious stimulus that might induce sympathetic responses. The purpose of this study, was to evaluate the effects of jaw-thrust on sympathetic responses.

Methods: We investigated seventy three patients. Patients who received general anesthesia were randomly divided into a control group (maintenance of combined airway maneuver with head tilt, open mouth by mouthpiece, and chin-lift, $\mathrm{n}$ = 30) and jaw-thrust group (maintenance of head tilt, open mouth and jaw-thrust, $\mathrm{n}=30$ ). In the jaw-thrust group, four minutes of endoscopy-guided force to the mandible to get the best laryngeal view were applied. For the control group, the combined airway maneuver was maintained during the same period. Arterial blood pressure (AP) and heart rate (HR) were recorded at predetermined time points ( $1 \mathrm{~min}$ before anesthesia induction, 2 min after fiberoptic bronchoscopy placement, and thereafter 1 min-interval during each airway maneuver) during jaw-thrust and chin-lift maneuver. The force amplitude applied for best laryngeal view during jaw-thrust was also measured.

Results: Peak systolic and diastolic AP increased $39.0 \pm 17.6$ and $39.9 \pm 22.8 \mathrm{mmHg}$ from the baseline $(\mathrm{P}<0.001)$ in the jaw-thrust group. HR was also $32.5 \pm 19.4$ beats/min from the baseline $(\mathrm{P}<0.001)$ in the jaw-thrust group. These remained high at all time points, compared with the control group $(\mathrm{P}<0.01)$. The force magnitude applied for jaw-thrust was not correlated to the AP and HR changes $(\mathrm{P}>0.05)$.

Conclusions: Performing the jaw-thrust maneuver induces significant sympathetic responses, irrespective of the force magnitude. (Korean J Anesthesiol 2013; 65: 127-131)

Key Words: Force, Jaw-thrust, Sympathetic.

Received: December 12, 2012. Revised: 1st, January 29, 2013; 2nd, January 31, 2013. Accepted: February 1, 2013.

Corresponding author: Dae-Lim Jee, M.D., Department of Anesthesiology and Pain Medicine, Yeungnam University Hospital, 317-1, Daemyungdong, Nam-gu, Daegu 705-035, Korea. Tel: 82-53-620-3364, Fax: 82-53-626-5275, E-mail: adjee@med.yu.ac.kr

(c) This is an open-access article distributed under the terms of the Creative Commons Attribution Non-Commercial License (http:// creativecommons.org/licenses/by-nc/3.0/), which permits unrestricted non-commercial use, distribution, and reproduction in any medium, provided the original work is properly cited. 


\section{Introduction}

During the induction of general anesthesia, loss of airway muscle tone makes the tongue and epiglottis move towards the pharyngeal wall, and result in the obstruction of the upper airway. The jaw-thrust maneuver is an effective technique to maintain a patent's airway in this situation. However, the jawthrust has been reported to cause jaw pain [1], discomfort [2], bruising [3], or patient motor reflex responses [4]. These observations may suggest that the jaw-thrust is a noxious stimulus that might induce sympathetic responses such as hypertension and tachycardia.

No previous report has specifically evaluated whether and how the jaw-thrust induces sympathetic responses. In this study, we investigated the magnitude of these sympathetic responses during the jaw-thrust.

\section{Materials and Methods}

After receiving approval from the Institutional Review Board and patients' written informed consent, we studied 73 American Society of Anesthesiologists 1 adult patients (aged 47-76 years), undergoing general anesthesia. Patients with hypertension, cardiovascular diseases, and cerebrovascular diseases were excluded.

Patients were not premedicated. Electrocardiogram (lead II), noninvasive blood pressure (NIBP), pulse oximetry $\left(\mathrm{SpO}_{2}\right)$, endtidal $\mathrm{CO}_{2}$ and sevoflurane concentration, and bispectral index (A-2000 bispectral index, Aspect Medical Systems, Leiden, Netherlands) were monitored. Blood pressure was measured at the patient's arm. Anesthesia was standardized for all patients. Anesthesia was induced with thiopental $(3 \mathrm{mg} / \mathrm{kg}$ intravenous [IV]) and sevoflurane (2.0-4.0 vol\%) in 50\% $\mathrm{N}_{2} \mathrm{O}$ in $\mathrm{O}_{2}$. Rocuronium ( $1 \mathrm{mg} / \mathrm{kg}$ IV) was used for muscle relaxation. The patient's lungs were ventilated using a Patil-Syracuse endoscopy mask attached to a circle system. End-tidal $\mathrm{CO}_{2}$ was kept between 32 and $37 \mathrm{mmHg}$ and the sevoflurane vaporizer was adjusted to achieve a stable BIS value of 40-60. After induction of anesthesia, the difficulty of positive pressure ventilation was assessed and graded as follows: grade 1, no difficulty; grade 2 , needs chin lift; grade 3 , needs oral airways or jaw-thrust to maintain airway patency. The patients in grade 3 were excluded from the study. Then all the patients received a combination of head tilt, open mouth (by inserting a mouthpiece between the upper and lower incisors) and chin-lift while holding the endoscopy mask with one hand, to facilitate the placement of the fiberoptic bronchoscope (11301BN bronchoscope, Karl Storz GmbH \& Co. KG, Tuttlingen, Germany) and to maintain adequate ventilation. Three minutes after the induction, the anesthesiologist advanced the tip of the fiberoptic bronchoscope through the hole of the endoscopy mask into the mouth down to the retrouvular region, where it remained in place for the remainder of the study period.

The patients were randomly divided into a control group (by performing nothing additional, i.e., the combination of head tilt, open mouth and chin-lift) or a jaw-thrust group (by performing jaw-thrust instead of chin-lift, i.e., the triple airway maneuver) using a computer-generated table of random numbers. In the jaw-thrust group, the anesthesiologist stood on the floor weighing scales with an instrument stand (Fig. 1). The thumbs held the mask and the other finger tips were placed under the rami of the mandible (near the angle) with the anesthesiologist's arms on the instrument stand. The patient's jaw was lifted vertically by lowering the operating table for 4 minutes. The operating table was lowered until the best endoscopic laryngeal view (i.e., full view of the arytenoids and glottis as described by Timmermann et al. [5]) was obtained with fiberoptic bronchoscopy (FOB).

FOB was used to guide the anesthesiologist to apply 'adequate' force to the mandible necessary for obtaining the best endoscopic laryngeal view during the jaw-thrust: for force that is above adequate, the jaw-thrust might result in exaggerated sympathetic responses while for force that is below, the best laryngeal view would not be obtained. Thus, endoscopy could help the anesthesiologist adjust the force, thereby avoiding the use of excessive or not enough force. In the control group, the tip of the FOB also remained in the same position for the same duration as in the jaw-thrust group. When the tip of the FOB could not be advanced behind the uvula without the jaw-

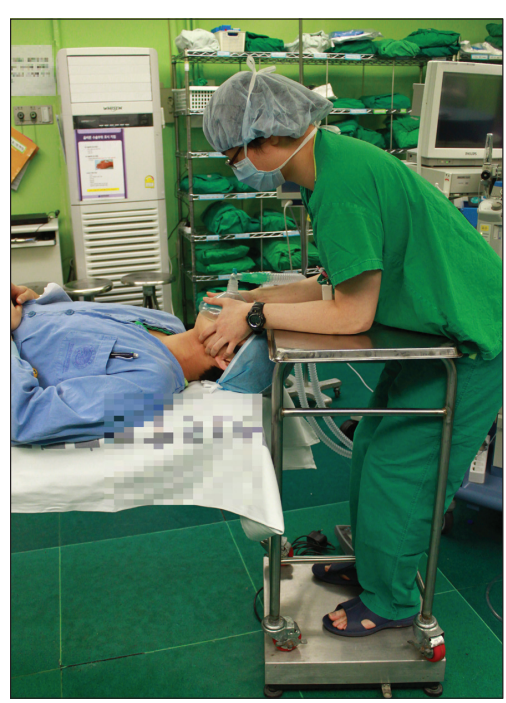

Fig. 1. The anesthesiologist stands on a floor weighing scale with an instrument stand in the jaw-thrust group. The thumbs held the mask and the other finger tips were placed under the rami of the mandible (near the angle) with the anesthesiologist's arms against the instrument stand. The patient's jaw was lifted vertically by lowering the operating table. 
thrust, the patient was excluded from the study. To ensure standardization, a single anesthesiologist performed the airway maneuver or endoscopy.

The force applied to the mandible was assessed using a weighing scale method for the cricoid force measurement, in which the downward force applied to the cricoid cartilage affects the measured body weight of the person applying it [6,7]. In our study, the anesthesiologist stood on a floor weighing scale equipped with an arm rest which reflected the steady upward force applied during the jaw-thrust to the floor weighing scale; the scale was placed below the head of the operating table. The vertical, steady force applied to the patient's mandible by the anesthesiologist was transmitted to the floor weighing scale. The kilogram value was converted to a unit of force $(1 \mathrm{~kg}=9.81$ N). The floor scale used (Ohaus CQ100-L31 Champ SQ Bench scale, Ohaus Corp, Pine Brook, USA) has a display indicator (model CD-31, Ohaus Corp, Pine Brook, USA), with a range of $0.000-100.000 \mathrm{~kg}$ and a resolution of $0.005 \mathrm{~kg}$. The forces were recorded every one minute during the jaw-thrust. Patients with a difference between the highest and lowest forces $\geq 1 \mathrm{~N}$ during the jaw-thrust were arbitrarily considered as having failed to maintain a steady force and excluded from the study.

Arterial pressure (AP) and heart rate (HR) were measured at the following time points: $1 \mathrm{~min}$ before the induction of anesthesia (baseline), and $5 \mathrm{~min}$ after anesthesia induction (prestimulation, i.e., 2 min after FOB placement), and thereafter, at 1 min-intervals during each airway maneuver (Fig. 2). To be more concrete, the non-invasive blood pressure measuring instrument began to operate taking blood pressure measurements and HR was recorded at these times. The peak AP and HR during the jaw-thrust were recorded. With the forces measured, we examined if the peak AP and HR were linearly correlated to the highest and lowest forces. The anesthesiologists who performed FOB were blind to the change of AP, HR, and weight scale. Another anesthesiologist who performed the jaw-thrust maneuver was also blind to the change of AP, HR, weight scale, and the endoscopic finding.

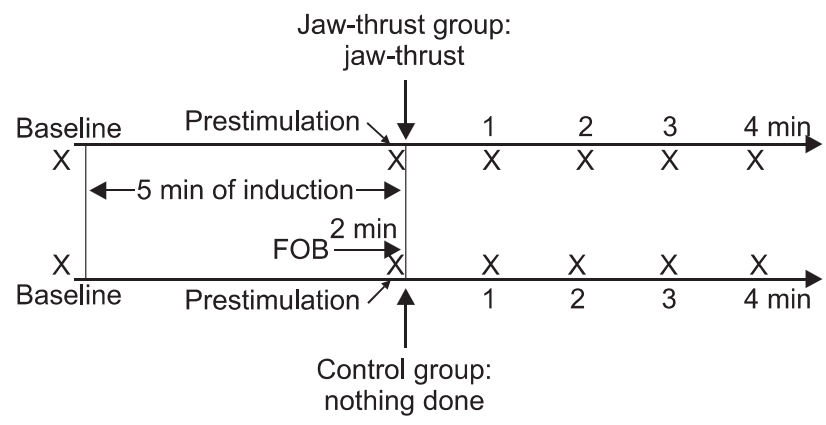

Fig. 2. Study protocol. FOB: flexible fiberoptic bronchoscopy, X: time point of arterial pressure, heart rate and force measurement.
The peak HR during the course of the measurements was considered as the primary outcome. Interim analysis showed that the sample size was calculated as 30 patients for each group to detect the difference in HR (15 beats/min) between the groups with a power of $90 \%$ and a type 1 error rate of 0.01 . Repeated measured ANOVA was used for AP and HR. A Pearson correlation coefficient was calculated to determine the correlation of AP and HR to the forces applied. The inter-group differences for patient characteristics were evaluated using unpaired t-test, $\chi^{2}$ test, and Mann Whitney U-test. P values of $<0.05$ were considered significant. Data were presented as the mean \pm SD or number.

\section{Results}

Of the 73 patients enrolled in this study, 60 completed the study. Thirteen patents were excluded from the analysis of the results for the following reasons: failure to place the tip of the bronchoscope into the retrouvular region ( 2 in the jaw-thrust group, 1 in the control group); difficult mask ventilation (2 in the jaw-thrust group, 1 in the control group); failure to maintain constant force during jaw-thrust (7 patients). No differences were found between the two groups with respect to the patient characteristics (Table 1).

The changes of AP (systolic and diastolic) and HR during each airway maneuver were compared with the baseline and between the groups (Fig. 3). The baseline values were similar in both groups. Systolic and diastolic AP and HR of the jaw-thrust

Table 1. Characteristics of the Patients

\begin{tabular}{lcc}
\hline & $\begin{array}{c}\text { Jaw-thrust group } \\
(\mathrm{n}=30)\end{array}$ & $\begin{array}{c}\text { Control group } \\
(\mathrm{n}=30)\end{array}$ \\
\hline Sex $(\mathrm{M} / \mathrm{F})$ & $13 / 17$ & $15 / 15$ \\
Age $(\mathrm{yr})$ & $59.3 \pm 8.8$ & $59.2 \pm 7.8$ \\
Weight $(\mathrm{kg})$ & $58.1 \pm 11.0$ & $61.7 \pm 9.6$ \\
Height $(\mathrm{cm})$ & $161.2 \pm 7.3$ & $161.5 \pm 8.5$ \\
BMI (kg/m $\left.{ }^{2}\right)$ & $22.2 \pm 2.9$ & $23.6 \pm 2.9$ \\
BIS & & \\
Preinduction & $94.3 \pm 3.9$ & $94.6 \pm 2.5$ \\
Prestimulation & $54.7 \pm 5.0$ & $55.6 \pm 3.8$ \\
$\mathrm{E}^{\prime}$ sevo $(\%)$ prestimulation & $1.86 \pm 0.31$ & $1.81 \pm 0.16$ \\
Grade of ventilatory difficulty (n) & & 8 \\
Grade 1 & 9 & 22 \\
Grade 2 & 21 & 0 \\
Grade 3 & 0 & \\
Baseline hemodynamics & & $721.3 \pm 9.0$ \\
SAP (mmHg) & $118.7 \pm 10.1$ & $73.8 \pm 4.5$ \\
DAP (mmHg) & $75.3 \pm 7.7$ & \\
HR (beats/min) & $75.0 \pm 6.7$ & \\
\hline
\end{tabular}

Data were expressed as the mean \pm SD or numbers. BMI: body mass index, BIS: bispectral index, Prestimulation: before performing jawthrust or nothing, $\mathrm{E}_{\text {sevo }}$ : end-tidal sevoflurane concentration, SAP: systolic arterial pressure, DAP: diastolic arterial pressure, HR: heart rate. 


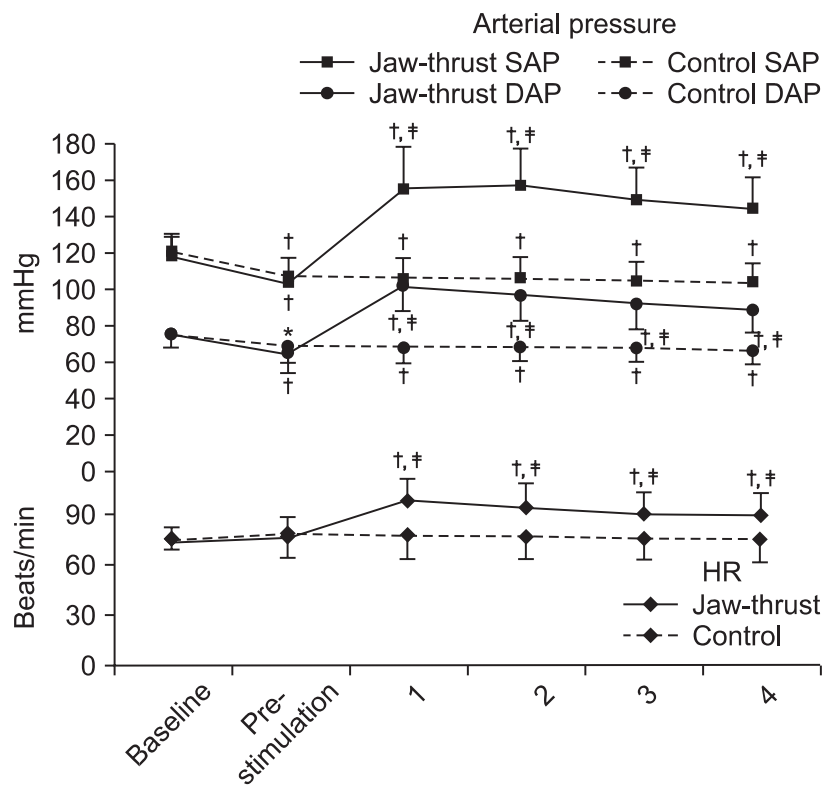

Fig. 3. Changes in arterial pressure and heart. Data were expressed as the mean $\pm \mathrm{SD} .{ }^{*} \mathrm{P}=0.009,{ }^{\dagger} \mathrm{P}<0.001$ when compared with the baseline and ${ }^{\dagger} \mathrm{P}<0.001$ when compared with the control group. SAP: systolic arterial pressure, DAP: diastolic arterial pressure, HR: heart rate. Baseline: 1 min before anesthesia induction. Prestimulation: before performing jaw-thrust or nothing. At 1, 2, 3, 4; 1, $2,3,4$ min after jawthrust.

group (systolic: $155.2 \pm 23.6,157.4 \pm 20.3,149.3 \pm 17.7,144.7 \pm$ $17.2 \mathrm{mmHg}$; diastolic: $102.5 \pm 14.3,97.3 \pm 14.1,92.4 \pm 13.9,89.3$ $\pm 12.3 \mathrm{mmHg}$; HR: $97.8 \pm 13.0,94.3 \pm 13.9,90.5 \pm 12.7,89.4$ \pm 13.3 beats $/ \mathrm{min}$ ) increased and remained high at all the time points during the jaw-thrust, compared to the baseline $(\mathrm{P}<$ 0.001 ) and those of the control group(systolic AP: $106.3 \pm 11.0$, $106.3 \pm 11.8,104.8 \pm 10.9,103.4 \pm 11.7$; diastolic AP: $68.4 \pm 8.7$, $68.5 \pm 7.8,67.9 \pm 7.9,66.7 \pm 8.0 \mathrm{mmHg}$; HR: $77.6 \pm 14.1,76.7$ $\pm 13.3,75.7 \pm 13.1,74.9 \pm 12.0$ beats $/ \mathrm{min})(\mathrm{P}<0.001)$. The jawthrust group also had an increase in the peak AP (systolic: 164.5 $\pm 21.9 \mathrm{mmHg}, 39.0 \pm 17.6 \%$; diastolic: $105.2 \pm 14.0 \mathrm{mmHg}, 39.9$ $\pm 22.8 \%)$ and HR $(98.8 \pm 12.6$ beats/min, $32.5 \pm 19.4 \%)$ from the baseline $(\mathrm{P}<0.001)$. There was no significant difference in the $\mathrm{AP}$ and $\mathrm{HR}$ at prestimulation between the groups. There was a modest decrease in systolic and diastolic AP $(\mathrm{P}<0.001$, respectively) but no significant change in $\mathrm{HR}$ at prestimulation, compared with the baseline in the jaw-thrust group. In the control group, AP decreased modestly whereas HR did not change, at all the time points compared with the baseline.

The mean intra-patient difference in adequate force was $0.77 \pm 0.25 \mathrm{~N}$ with a maximum of 0.98 and a minimum of $0.49 \mathrm{~N}$. The mean value of the highest forces in the intrapatient difference in adequate force was $34.07 \pm 9.33 \mathrm{~N}$ with a maximum of 59.35 and a minimum of $19.13 \mathrm{~N}$. The mean value of the lowest forces in the intra-patient difference in adequate force was $33.31 \pm 9.30 \mathrm{~N}$ with a maximum of 58.37 and a minimum of $18.15 \mathrm{~N}$ during the jaw-thrust. The magnitude of the force applied to the mandible was not linearly correlated to the degree of the increase in AP and HR from the baseline in the jaw-thrust group (Pearson's $r=-0.186,-0.339$, and -0.268 diastolic and diastolic AP and HR; each P > 0.05).

\section{Discussion}

The jaw-thrust maneuver is commonly performed for several minutes, to help optimize the condition for fiberoptic bronchoscopic intubation or to overcome airway obstruction in the operating room, intensive care unit, or emergency department. In this study, the jaw-thrust with endoscopy-guided adequate force application to the patients with BIS of 40-60 caused a significant rise in systemic AP and HR more than was expected. In addition, the inter-patient range for adequate force was wide and the sympathetic responses were not related to the magnitude of the adequate force applied.

It is not clear why the jaw-thrust can cause sympathetic responses. From our practice, we know painful stimulation from the jaw-thrust may be the cause. Plausibly, the jaw-lift maneuver, which is suggested to cause circulatory responses and has the same airway clearing effects on the upper airway as the jaw-thrust maneuver [8], may yield a clue to the sympathetic activation: the jaw-thrust widens the pharyngeal space and leads to motion of the pharynx and larynx and may stretch the mucosa of the pharynx and larynx. This mechanical stimulation to the mucosa may activate rapidly adapting receptors (RARs) within the pharynx and larynx, causing the sympathetic responses [9]. The RARs may readily be recruited by rapid changes in airway volume and intraluminal mechanical stimuli [10]. The activation of RARs may be another mechanism for the sympathetic responses.

It is difficult to explain why the sympathetic responses are irrelevant to the force. Pain cannot fully explain it. If painful stimulation from the jaw-thrust is the main mechanism for the sympathetic responses and pain is proportional to force applied, higher force would evoke more intense responses. However, in the present study, the sympathetic responses were not related to the magnitude of the adequate force applied. On the one hand, activation of RARs would induce the sympathetic responses as described above, and any force above the threshold that could result in the RARs activation might produce a similar intensity of sympathetic responses, irrespective of the magnitude of the force. We postulate that this may also partly explain the reason.

Some studies have reported on the contradictory effects of jaw-thrust on AP and HR. Arai et al. [11] reported that inhaled anesthesia significantly increased the stridor score and the sympathetic activity in the neutral neck position, while jaw- 
thrust relieved the occlusion of the airway and decreased the excited sympathetic nerve activity. However, there was no mention of the physical stimulation from jaw-thrust causing sympathetic activation. Stacey et al. [12] also reported that jawthrust or laryngoscopy for fiberoptic intubation did not evoke sympathetic responses; however, leaving the effects of jawthrust aside, laryngoscopy itself has been well known to cause hypertension and tachycardia, differently from the results of Stacey's study. In our opinion, jaw-thrust can stimulate the patient in a way inducing pain or a reflex response by physical force. Postoperative jaw pain [1], discomfort [2], bruising [3], or patient motor reflex responses are all evidence of such stimulation [4]. Thus, we postulate that lower forces might have been applied in those studies [11,12]. Although lower forces are not enough to obtain the best laryngeal view, they can open the airway and relieve sympathetic activation, or help perform fiberoptic intubation. In addition, application of the jaw-thrust was shorter in these studies than in our study. The shorter the duration of the jaw-thrust is, the lower the intensity of the sympathetic responses might be, because applied force is not enough to cause sympathetic stimulation. These may be responsible for the discrepancy.

The sympathetic responses of the jaw-thrust maneuver may be transient, but there can be clinical significance depending on the patient's condition such as hypertension, cardiovascular diseases and cerebrovascular diseases. Thus, clinicians should be alert to the possibility for exaggerated sympathetic responses to jaw-thrust. In such at risk patients, it would be occasionally necessary to blunt the responses in patients, using opioids (in the case of painful stimulation) or vasodilators/beta-blockers (in the case of activation of RARs).

A floor scale has been used for a force-measuring method during Sellick's maneuver $[6,7,13]$. In the present study an upward force, instead of the downward force for Sellick's maneuver, was applied to the rami of the mandible and the change in body weight of the anesthesiologist was measured. Because the intrapatient difference of the force was narrow $(0.77 \pm 0.25 \mathrm{~N})$, using this method seemed to be appropriate and precise in measuring the force applied to the mandible during the total period of the jaw-thrust.

In summary, performing the jaw-thrust maneuver can induce significant sympathetic responses. The responses are not related to the magnitude of the applied force. Clinicians should be alert to the possibility for sympathetic responses to the jaw-thrust maneuver.

\section{References}

1. Brimacombe J, Holyoake L, Keller C, Brimacombe N, Scully M, Barry J, et al. Pharyngolaryngeal, neck, and jaw discomfort after anesthesia with the face mask and laryngeal mask airway at high and low cuff volumes in males and females. Anesthesiology 2000; 93: 26-31.

2. Reber A, Paganoni R, Frei FJ. Effect of common airway manoeuvres on upper airway dimensions and clinical signs in anaesthetized, spontaneously breathing children. Br J Anaesth 2001; 86: 217-22.

3. Nicoll SJ. An unusual complication of a jaw thrust. Anaesthesia 2007; 62: 423.

4. Drage MP, Nunez J, Vaughan RS, Asai T. Jaw thrusting as a clinical test to assess the adequate depth of anaesthesia for insertion of the laryngeal mask. Anaesthesia 1996; 51: 1167-70.

5. Timmermann A, Russo S, Graf BM. Evaluation of the CTrach - an intubating LMA with integrated fibreoptic system. Br J Anaesth 2006; 96 : 516-21.

6. Clayton TJ, Vanner RG. A novel method of measuring cricoid force. Anaesthesia 2002; 57: 326-9.

7. Haslam N, Parker L, Duggan JE. Effect of cricoid pressure on the view at laryngoscopy. Anaesthesia 2005; 60: 41-7.

8. Hirabayashi Y, Hiruta M, Kawakami T, Inoue S, Fukuda H, Saitoh K, et al. Effects of lightwand (Trachlight) compared with direct laryngoscopy on circulatory responses to tracheal intubation. Br J Anaesth 1998; 81: 253-5.

9. Nishino T, Tagaito Y, Isono S. Cough and other reflexes on irritation of airway mucosa in man. Pulm Pharmacol 1996; 9: $285-92$.

10. Sant'Ambrogio G, Widdicombe J. Reflexes from airway rapidly adapting receptors. Respir Physiol 2001; 125: 33-45.

11. Arai YC, Nakayama M, Kato N, Wakao Y, Ito H, Komatsu T. The effects of jaw thrust and the lateral position on heart rate variability in anesthetized children with obstructive sleep apnea syndrome. Anesth Analg 2007; 104: 1352-5.

12. Stacey MR, Rassam S, Sivasankar R, Hall JE, Latto IP. A comparison of direct laryngoscopy and jaw thrust to aid fibreoptic intubation. Anaesthesia 2005; 60: 445-8.

13. Clark RK, Trethewy CE. Assessment of cricoid pressure application by emergency department staff. Emerg Med Australas 2005; $17: 376-81$. 